\title{
Source Localization and Tracking in a Cognitive Radio Environment Consisting of Frequency and Spatial Mobility
}

\author{
Agur Adams \\ Department of Electrical and Computer Engineering \\ United States Naval Academy \\ Annapolis, United States of America \\ aadams@usna.edu
}

\author{
Murali Tummala, John McEachen, and James Scrofani \\ Department of Electrical and Computer Engineering \\ Naval Postgraduate School \\ Monterey, United States of America \\ $\{$ mtummala, mceachen, jwscrofa $\} @$ nps.edu
}

\begin{abstract}
Source localization and tracking of a Cognitive Radio (CR) is a significant challenge because of the dynamic opportunistic behavior of the radio across the spatial, frequency, and temporal domains. For any localization or tracking scheme to be effective, it must be able to adapt as a CR adapts to its surroundings. The extended semi range-based (ESRB) localization scheme was proposed as a solution to the aforementioned problem, but resulted in considerable communication overhead and storage requirements within the wireless sensor network (WSN) as well as poor reliability due to frequent divergence of the non-linear least squares method (NLSM) in the localization process. Furthermore, tracking a mobile $\mathrm{CR}$ was accomplished in a brute force manner by repeating the same positioning technique without taking advantage of prior position estimates. In this paper, the ESRB localization scheme is modified to incorporate the Kalman filter as a recursive estimator to reduce the burden placed on the WSN and integrate an efficient means to estimate the position and velocity of a mobile CR over time. The proposed modification is modeled in the MATLAB programming language, and its efficacy is demonstrated through simulation. It is shown that the Kalman filter is an appropriate recursive estimator for use in the ESRB localization scheme, while accounting for both frequency and spatial mobility inherent in the CR environment.
\end{abstract}

\section{INTRODUCTION}

A clear tension remains between the perceived shortage of the electromagnetic spectrum and the poor efficiency in its usage around the world [1]. Cognitive Radio (CR) technology has been considered the optimal means to resolve this conflict by enabling a radio to operate in the unused or underutilized portions of the electromagnetic spectrum (i.e., 'white spaces') [1]. Such opportunistic behavior is possible by two fundamental attributes of CR technology: 1) selfawareness through spectrum sensing, and 2) self-adaptation through autonomous decision making [1]. These advantages are being invested in heavily by the U.S. Department of Defense (DoD) (e.g., dynamic spectrum access in the Joint Tactical Radio System (JTRS) [2]) and the commercial market (e.g., carrier aggregation in LTE-Advanced [3]) for use in new radio systems. Given the increasing demand for security in mobile wireless devices, it is critical to begin examining the impact of source localization and tracking on CR technology

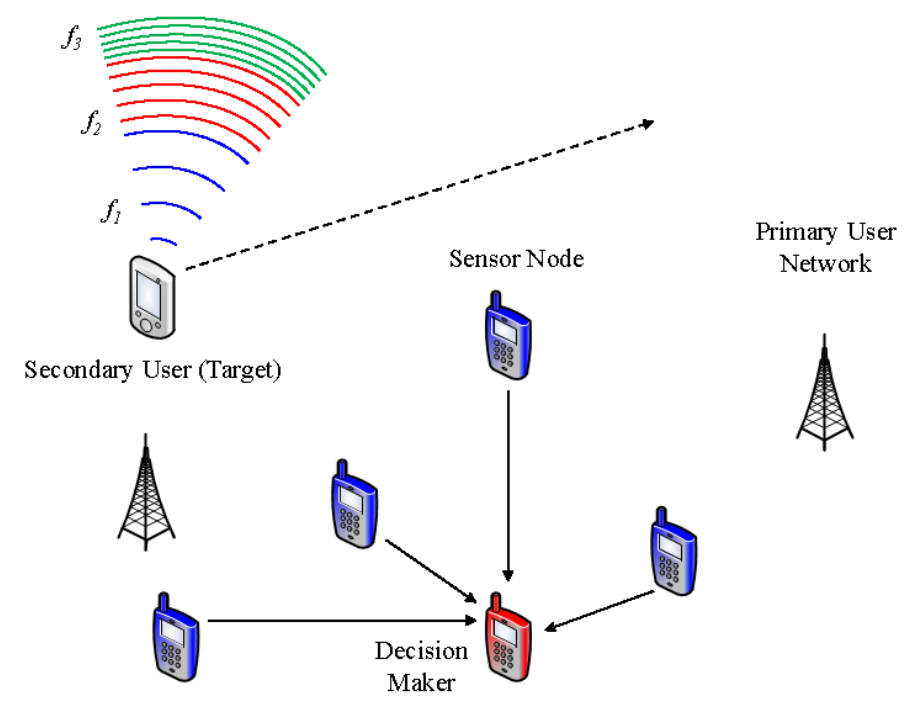

Fig. 1. Overall scenario using a wireless radio frequency sensor network to determine the frequency bands and location of a mobile cognitive radio.

before it ubiquitously prevails as an integral part of modern communication systems.

The adoption of CR technology is motivated by its efficient integration with existing communication systems. As shown in Fig. 1, two types of networks are assumed present: the network of primary users (PU), and the network of secondary users (SU). The PU network, typically some form of legacy communication system, has complete access to the assigned spectrum and offers no assistance to any other type of user in the environment. Conversely, the SU relies on opportunistically utilizing unused PU network resources without interfering with the PU's operations.

The extended semi range-based (ESRB) localization scheme, originally introduced in [1], offers a promising solution to the problem of estimating a CR's location in such an environment. As depicted in Fig. 1, the scheme utilizes a wireless radio frequency sensor network (WSN) for localization, taking advantage of the sensing diversity gain achieved 
through cooperative spectrum sensing. However, it is not optimal when tracking a CR's position over time in three critical ways. First, implicit in the ESRB localization scheme is the faultless communication between sensor nodes and the use of unlimited resources by a Decision Maker (DM) to record the heavy volume spectrum sensing results necessary to identify the SU. Second, localization through the non-linear least squares method (NLSM) frequently diverges resulting in an inaccurate position estimate of the SU. Finally, it does not employ a method to take advantage of previous position estimates as the target moves within the environment. Thus, a recursive estimation technique is needed to 1) reduce the volume of communication data and storage in the WSN, 2) overcome the margin of error produced by the NLSM, and 3) take advantage of past localization estimates. This paper proposes the Kalman filter as a viable recursive estimator to overcome these shortfalls of the ESRB scheme.

Section II gives the methodology and shortfalls of the ESRB localization scheme. Section III presents the integration of the Kalman filter into the ESRB localization scheme. Section IV explicates the simulation scenario and model used to implement the modified ESRB localization scheme and the simulation results; and finally, Section V summarizes the work completed and the significant results.

\section{Methodology And Shortfalls of The EXTEnded SEMI RANGE-BASED LOCALIZATION SCHEME FOR Cognitive Radio Positioning And TRACKInG}

The ESRB localization scheme's central notion is to determine the user's position in the spatial domain by making use of the PU's or SU's spectral occupancy pattern in the frequency domain [1]. Distinguishing between the two types of users is solely a function of a user's position at a point in time. The assumption is that the positions of all PUs are available through a geo-location database, as in the IEEE 802.22 standard [4]-[6]. Therefore, any user localized outside of the PU's known location is assumed to be a SU. The ESRB localization scheme is summarized in the flow diagram of Fig. 2, which begins with spectrum sensing at the sensor nodes of the WSN. [1]

For simplicity and speed, energy based detection is performed by the $i$ th sensor node in a channel $k$ of fixed bandwidth $W$ for a period $T$ to distinguish one of two hypotheses

$$
x_{i}(t)=\left\{\begin{array}{rl}
n(t), & H_{0} \\
s_{i}(t)+n(t), & H_{1}
\end{array} \quad 0<t \leq T\right.
$$

where $x_{i}(t)$ is the received signal, $s_{i}(t)$ is the signal of interest from the user occupying the channel, and $n(t)$ is Gaussian noise with zero mean and standard deviation $\sigma_{n}$. Assuming multipath fading, path loss, and shadowing as in [1], the average SNR at each node from a user at distance $d_{i}$ transmitting at power $P_{t x}$ is

$$
\overline{\gamma_{i}}=\frac{\overline{\left(s_{i}\right)^{2}}}{\sigma_{n}^{2}}=\frac{c P_{t x}\left(d_{i}\right)^{-\alpha}}{\sigma_{n}^{2}}
$$

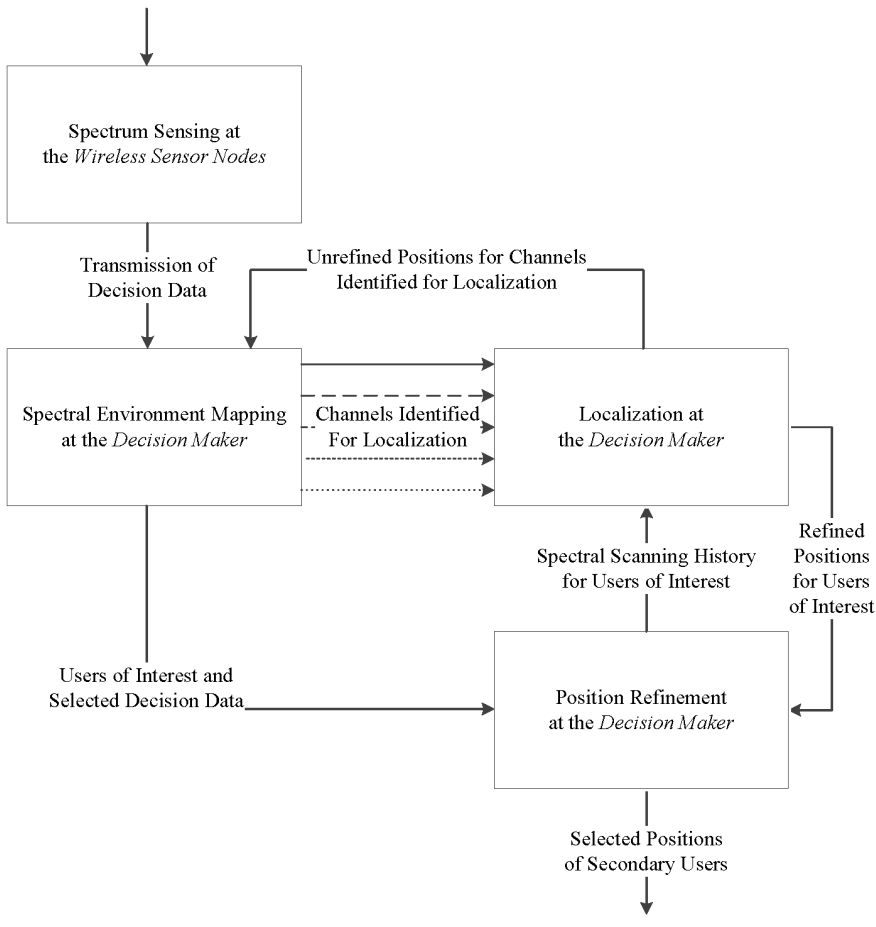

Fig. 2. Conceptual diagram of the extended semi range-based (ESRB) localization scheme for cognitive radio positioning.

where $\alpha$ represents the path loss factor and $S$ the shadowing effect which follows a log-normal distribution. As shown in [7], the probability of false alarm $\overline{P_{f}}$ and probability of detection $\overline{P_{d}}$ under these conditions are

$$
\begin{aligned}
\overline{P_{f, i}}= & \mathbb{E}\left[P\left\{X_{i}>\lambda_{i} \mid H_{0}\right\}\right]=\frac{\Gamma\left(u, \frac{\lambda_{i}}{2}\right)}{\Gamma(u)} \\
\overline{P_{d, i}}= & \mathbb{E}\left[P\left\{X_{i}>\lambda_{i} \mid H_{1}\right\}\right] \\
= & e^{-\frac{\lambda_{i}}{2}} \sum_{n=0}^{u-2} \frac{1}{n !}\left(\frac{\lambda_{i}}{2}\right)^{n}+\left(\frac{1+\overline{\gamma_{i}}}{\overline{\gamma_{i}}}\right)^{u-1} \\
& \times\left[e^{-\frac{\lambda_{i}}{2\left(1+\overline{\lambda_{i}}\right)}}-e^{-\frac{\lambda_{i}}{2}} \sum_{n=0}^{u-2} \frac{1}{n !}\left(\frac{\lambda_{i} \overline{\gamma_{i}}}{2\left(1+\overline{\gamma_{i}}\right)}\right)^{n}\right]
\end{aligned}
$$

where $\lambda_{i}$ is the energy detection threshold, $\Gamma(\cdot)$ the gamma function, and $\Gamma(\cdot, \cdot)$ the incomplete gamma function.

The essence of semi-range based localization is to use an estimate of $\overline{P_{d, i}}$ to determine distance from the known sensor node positions to the user [8]. By establishing $\overline{P_{f, i}}$ in advance, an optimal $\lambda$ can be derived from (3), which reduces (4) to a function of $u$ and the $\lambda_{i}$ [8]. The design of the WSN allows for a known $u$ further reducing (4) to strictly a function of $\lambda_{i}$, which can be translated into distance by (2) as shown in [8]. Estimating $\overline{P_{d, i}}$ is the critical factor. To solve this problem, the presence or absence of a user in a channel is communicated as a binary one or zero by each sensor node. Using a majority rule, the DM establishes a global result for the entire WSN that can be used to determine the accuracy of each individual sensor [7]. The $\overline{P_{d, i}}$ is estimated by how frequently a sensor's reported spectrum sensing results match the global result, 
TABLE I

THRESHOLDS AND FALSE ALARM VALUES FOR $n$-BIT SPECTRUM SENSING (FROM [9])

\begin{tabular}{|c|c|}
\hline Threshold & $\overline{P_{f}}$, False Alarm \\
\hline$\lambda_{i, 2^{n}-1}$ & $\beta_{2^{n}-1} \overline{P_{f}}$ \\
\hline$\ldots$ & $\ldots$ \\
\hline$\lambda_{i, 5}$ & $\beta_{5} \overline{P_{f}}$ \\
\hline$\lambda_{i, 4}$ & $\beta_{4} \overline{P_{f}}$ \\
\hline$\lambda_{i, 3}$ & $\beta_{3} \overline{P_{f}}$ \\
\hline$\lambda_{i, 2}$ & $\beta_{2} \overline{P_{f}}$ \\
\hline$\lambda_{i, 1}$ & $\beta_{1} \overline{P_{f}}$ \\
\hline
\end{tabular}

which can be repeated for all channels of interest [7]. In this way, the DM can develop a spectral environment map for all channels provided the history of all individual sensor measurements is maintained. This requirement places a heavy memory burden on the DM as the number of sensor nodes increases along with the duration of their operation. Those channels which are occupied are identified for localization, which occurs through the NLSM as follows.

To solve for the position of the user occupying channel $k$ using only the spectral scanning decision data available, (2) is rewritten as a function of $\boldsymbol{\theta} \triangleq\left[x, y, P_{t x}\right]$ as [7]

$$
\begin{aligned}
f_{i}(\boldsymbol{\theta})= & (x)^{2}-2 a_{i} x+(y)^{2}-2 b_{i} y+a_{i}^{2}+b_{i}^{2} \\
& -\left(\frac{c}{\overline{\gamma_{i}} \times \sigma_{n}^{2}}\right)^{\frac{2}{\alpha}} \times\left(P_{t x}\right)^{\frac{2}{\alpha}}
\end{aligned}
$$

where $x$ and $y$ are the Cartesian position coordinates of the user and $a_{i}$ and $b_{i}$ are the Cartesian position coordinates of the sensor. The estimate $\hat{\boldsymbol{\theta}}$ can be solved iteratively through the NLSM by computing the Gauss-Newton direction $\psi$ and the scalar $\kappa$ after an initial value for $\psi$ is obtained by

$$
\hat{\boldsymbol{\theta}}_{l+1}=\hat{\boldsymbol{\theta}}_{l}+\kappa \boldsymbol{\psi} .
$$

The Gauss-Newton direction $\psi$ and the scalar $\kappa$ provide a correction throughout each iteration of the NLSM [10]. Their derivation is shown in [7] for application in semi rangebased localization to achieve the minimum mean square error. Provided the initial estimate $\hat{\boldsymbol{\theta}}_{0}$ is close to the true solution, the NLSM will converge toward the local solution with each correction; however, the NLSM can and will fail when the initial estimate is not close. The failure is due either to the Gauss-Newton direction not being in a direction of descent or the length of the Gauss-Newton direction being too great [10]. This results in an increase of (6) and in a divergence of the iteration [10]. When this occurs, a solution cannot be obtained.

To reduce the frequency of divergence in the localization process, $n$-bit spectrum sensing can be employed. The use of additional bits increases the resolution of the sensor node's measurements and produces a more accurate estimate of $\overline{P_{d, i}}$ with fewer sensing measurements. This leads to a better estimate of the distance between the user and the sensor node, which allows the iterative NLSM to converge regularly. As

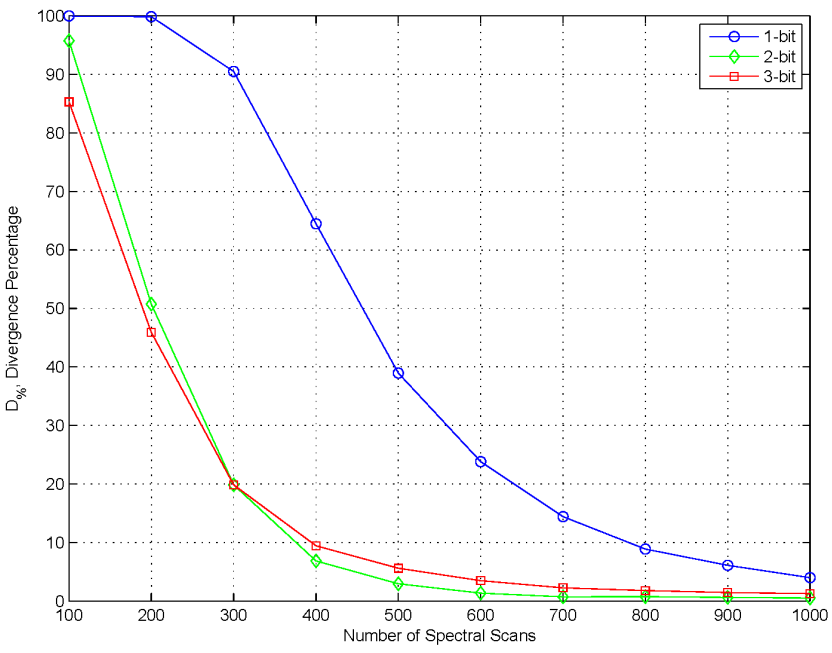

Fig. 3. Divergence percentage versus the number of spectral scans for three different bit values in $n$-bit spectrum sensing.

in two-bit [11] and three-bit [9] hard combination, multiple energy detection thresholds are established to create multiple energy regions [9], [11]. These thresholds are determined using Neyman-Pearson criterion [9], [11], where a universal $\overline{P_{f}}$ is declared for all sensor nodes and then scaled by the coefficients $\beta_{n}$ for each threshold as in Table I. These coefficients are determined by

$$
\beta_{n}=10^{-n} \quad n=2,3, \ldots, 2^{n}-1
$$

where $n$ is the threshold index and $\beta_{0}=1$ [9]. As shown in [9], determining thresholds are design issues.

The benefits of using $n$-bit spectrum sensing in the localization process are shown in are shown in Fig. 3. The divergence percentage for binary (i.e., 1-bit) spectrum sensing declines exponentially as the number of spectral scans increases linearly. However, using two-bit and three-bit spectrum sensing, the divergence percentage declines at an accelerated rate relative to binary spectrum sensing. Such acceleration indicates with higher bit-order spectrum sensing the number of localization attempts with divergence decreases as the bit-order increases, but it does not eliminate the problem completely.

Compounding the difficulty in resolving the position of the user occupying the channel, the position estimate is not often accurate through the NLSM when limited spectral scanning data is available as shown in [1]. When the SU is identified occupying different portions of the frequency spectrum the DM must pair past spectrum sensing decision data with current measurements to gain sufficient information for accurately estimating their position. Thus, the DM is forced to accrue measurement data from the WSN in order to refine the positions of users of interest. Given that the sensor nodes are potentially scanning numerous channels indefinitely; such actions are prohibitive due to the excessive amounts of memory and processing needed to synthesize this information. 


\section{InTEGRATION OF THE KALMAN FILTER INTO THE} EXTENDED SEMI RANGe-BASEd Localization Scheme FOR COGNITIVE RADIO POSITIONING AND TRACKING

To alleviate the communication and storage burden required from the WSN and the DM, recursive estimation can be used to reduce the past position estimates and spectral scanning data into a single predication of the target's trajectory. As shown in Fig. 4, a recursive estimator is applied to the existing ESRB scheme by taking the position estimates of the CR from the position refinement process every $T_{s}$ seconds. In this way, the WSN solely acts in a sensing function while the DM provides position estimation over time.

\section{A. State Space Model}

Assuming the target moves at a near constant velocity in a two dimensional Cartesian plane, its state can be defined as

$$
\mathbf{x}(n) \triangleq\left[\begin{array}{c}
x_{1}(n) \\
x_{2}(n) \\
x_{3}(n) \\
x_{4}(n)
\end{array}\right]
$$

where $x_{1}(n)$ and $x_{3}(n)$ are the target's horizontal and vertical position coordinates at time $n T_{s}$ and $x_{2}(n)$ and $x_{4}(n)$ are the target's horizontal and vertical velocity at time $n T_{s}$, respectively. The target's state space model is given by

$$
\begin{aligned}
\mathbf{x}(n+1) & =\mathbf{A} \mathbf{x}(n)+\mathbf{v}(n) \\
\mathbf{y}(n) & =\mathbf{C x}(n)+\mathbf{w}(n)
\end{aligned}
$$

where

$$
\begin{gathered}
\mathbf{A}=\left[\begin{array}{cccc}
1 & T_{s} & 0 & 0 \\
0 & 1 & 0 & 0 \\
0 & 0 & 1 & T_{s} \\
1 & T_{s} & 0 & 1
\end{array}\right], \mathbf{C}=\left[\begin{array}{cccc}
1 & 0 & 0 & 0 \\
0 & 0 & 1 & 0
\end{array}\right] \\
\mathbf{v}(n)=\left[\begin{array}{l}
v_{1}(n) \\
v_{2}(n) \\
v_{3}(n) \\
v_{4}(n)
\end{array}\right] \quad, \quad \mathbf{w}(n)=\left[\begin{array}{l}
w_{1}(n) \\
w_{2}(n)
\end{array}\right]
\end{gathered}
$$

with the vector $\mathbf{v}(n)$ representing the system noise, $\mathbf{w}(n)$ the measurement noise, and $\mathbf{y}(n)$ the position estimate every $T_{s}$ seconds from the ESRB localization scheme.

\section{B. The Kalman Filter}

The well-known Kalman filter is given by the following equations for predicting and correcting the estimate of the target's position and velocity [12]

$$
\begin{aligned}
\hat{\mathbf{x}}(n+1) & =\mathbf{A} \hat{\mathbf{x}}(n)+\overline{\mathbf{K}}(n)(\mathbf{y}(n)-\mathbf{C} \hat{\mathbf{x}}(n)) \\
\hat{\mathbf{P}}(n+1) & =\mathbf{A} \hat{\mathbf{P}}(n) \mathbf{A}^{T}-\overline{\mathbf{K}}(n) \mathbf{C} \hat{\mathbf{P}}(n) \mathbf{A}^{T}+\mathbf{Q}
\end{aligned}
$$

where

$$
\overline{\mathbf{K}}(n)=\mathbf{A} \hat{\mathbf{P}}(n) \mathbf{C}^{T}\left(\mathbf{C P}(n) \mathbf{C}^{T}+\mathbf{R}\right)^{-1}
$$

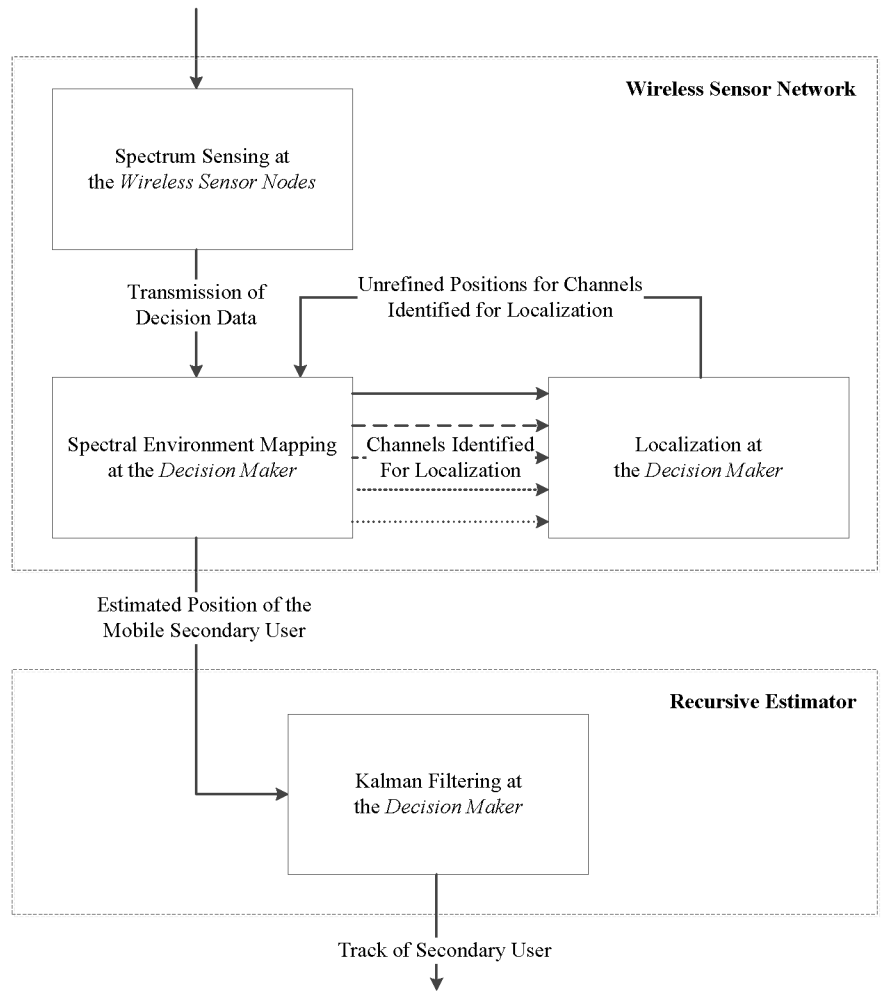

Fig. 4. Conceptual diagram of the modified extended semi range-based (ESRB) localization scheme for cognitive radio positioning.

and $\mathbf{R}=\mathbb{E}\left[\mathbf{w} \mathbf{w}^{T}\right]$ and $\mathbf{Q}=\mathbb{E}\left[\mathbf{v} \mathbf{v}^{T}\right]$. In the event the WSN is not able to derive the position of the CR, the DM uses the previous state estimate until an accurate observation can be obtained. That is, (11) and (12) reduce to

$$
\begin{aligned}
\hat{\mathbf{x}}(n+1) & =\hat{\mathbf{x}}(n) \\
\hat{\mathbf{P}}(n+1) & =\hat{\mathbf{P}}(n)
\end{aligned}
$$

\section{Simulation Model and Results}

The simulation model used to demonstrate the effectiveness of the modification to the ESRB localization scheme is depicted in Fig. 5. A mobile CR begins at $(-2000,1000)$ meters and moves approximately eight kilometers with a near constant velocity in a random path around the WSN, which is indicated by the solid blue line. The position is varied in both directions by a normal distribution with a standard deviation of 150 meters. In the figure, five stationary PUs are located at various points around the SU and designated by green squares. At the center of the environment is the WSN that is used to localize the mobile SU. Fifty sensor nodes, marked as red $X$ 's in the figure, are confined to a 900 meter perimeter and uniformly distributed throughout. Lastly, the DM is located at the origin. 


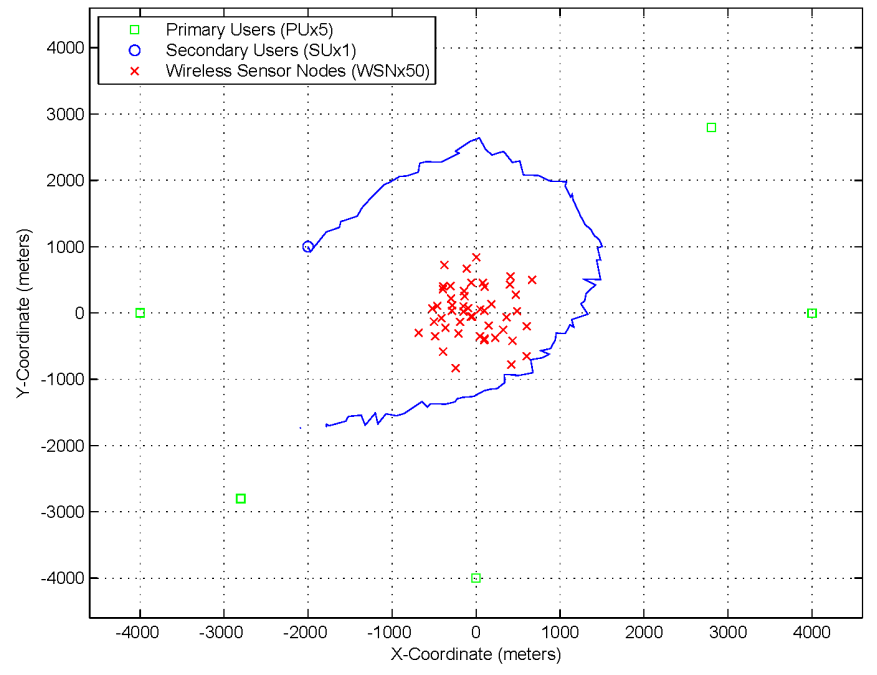

Fig. 5. Simulation model using a wireless radio frequency sensor network to determine the frequency bands and location of a mobile cognitive radio.

The initial uncertainty in the target's position and velocity is characterized by the matrix $\mathbf{P}$, which is set to the square of the maximum range the WSN is capable of receiving (5000 meters) and to the square of the maximum velocity the target may be moving ( 50 meters per second). The statistics of the system noise $\sigma_{v}$ and the measurement noise $\sigma_{w}$ are known $a$ priori to have a standard deviation of two meters per second and 250 meters, respectively.

The frequency spectrum is broken up into five nonoverlapping channels that each PU occupies at random intervals in accordance with a two-state Markov model. When in the 'busy' state, the PU transmits continuously at 18 Watts, but leaves the channel completely free during the 'idle' state. The occurrence of each state is controlled with the probabilities $p_{i}$ and $p_{b}$, respectively, both set to 0.3 . The mobile CR transmits continuously at 17 Watts in the white spaces left by the PU. The SU also introduces quiet periods during transmission to conduct fast and fine spectrum sensing in accordance with IEEE 802.22 [13], [14]. The SU will determine if the channel is occupied after fast and fine sensing has occurred over the course of one superframe. This prohibits the SU from occupying any channel for less than one superframe, and therefore is the most discrete unit of time in which its behavior can change. The $\overline{P_{f}}$ is set to 0.01 with $u$ equal to 5 as a performance requirement for the WSN, which drives the $\lambda$ to 13.96 by (3).

The channel is considered time-invariant during sensing by the WSN, but each sensor node suffers from multipath fading, shadowing, and path loss in reception. Specifically, path loss is modeled by (2) with $c$ set to $0.01, \alpha$ at 3 , and $\sigma_{n}$ at 1.41. The assumption is made that the WSN is capable of performing 300 scans within one superframe over 300 ms. The Kalman filter is given a position estimate after ten superframes (i.e., $T_{s}$ is three seconds) to allow for limited position refinement before the recursive estimator is updated.

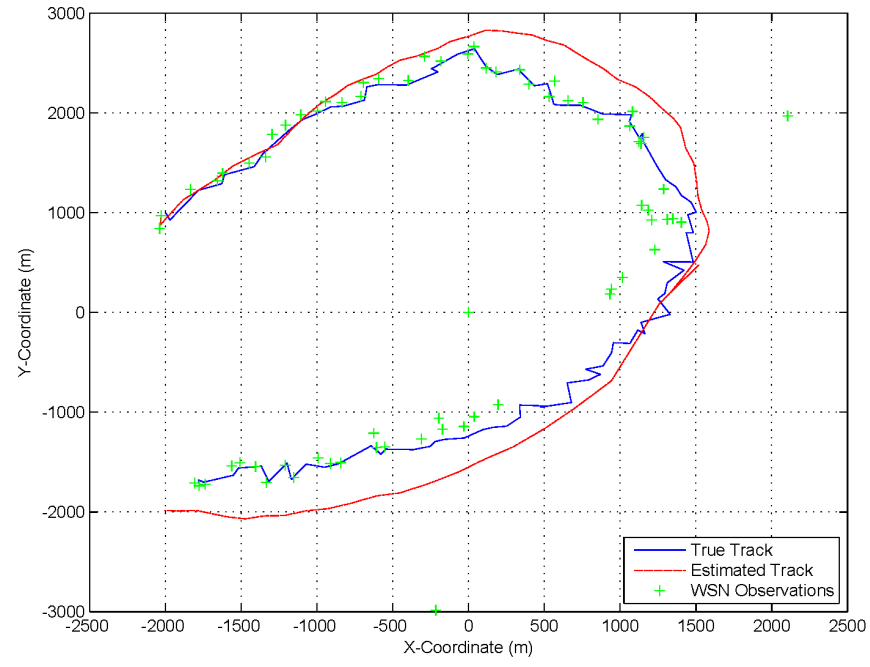

Fig. 6. Estimated and true trajectories of a mobile cognitive radio using a Kalman filter with the ESRB localization scheme in the two-dimensional Cartesian plane.

The integration of the Kalman filter into the ESRB localization scheme improves the ability to estimate a CR's position over time as indicated by the estimated and true trajectories shown in Fig. 6. Using the position estimates from the WSN, the DM is able to accurately track the position of the mobile CR in its random path around the WSN despite multiple occurrences of divergence in the NLSM. The frequency of divergence can be seen in Fig. 7 between iteration 40 and 70 of the Kalman filter. The WSN position estimates are near zero for almost every localization attempt during this period, yet the track by the Kalman filter still approximates the mobile CRs true position. The impact of divergence is more easily observed in the error in the estimated trajectory which is shown in Fig. 8.
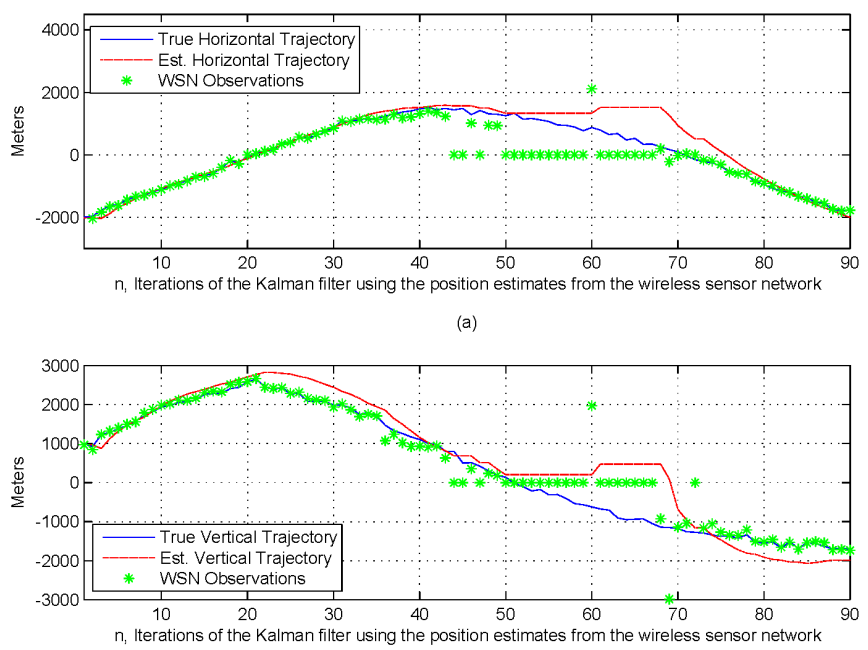

(b)

Fig. 7. Estimated and true horizontal and vertical trajectories of a mobile cognitive radio using a Kalman filter with the ESRB localization scheme: (a) estimated and true horizontal trajectories, (b) estimated and true vertical trajectory. 


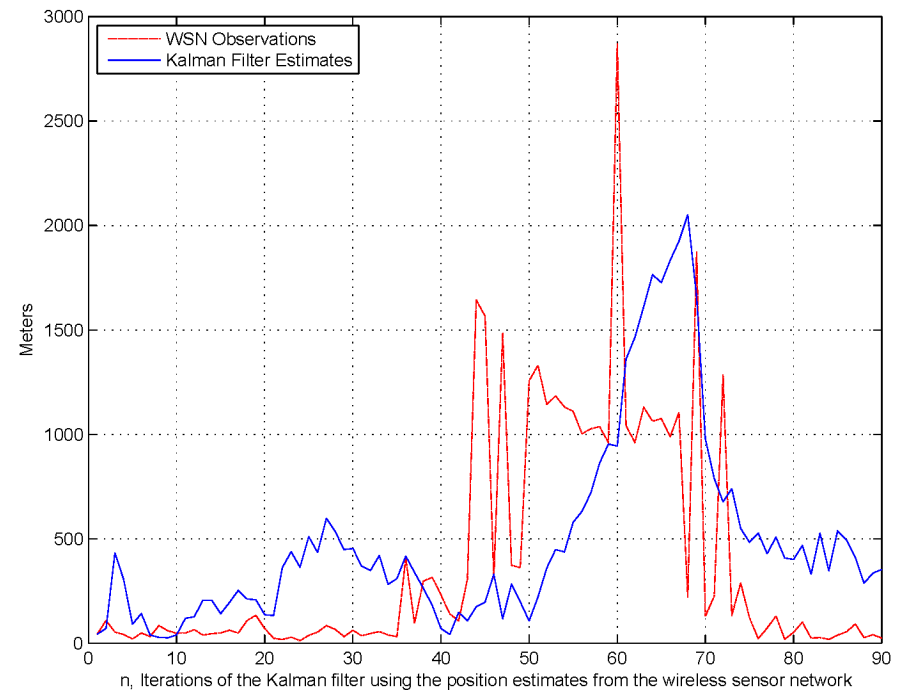

Fig. 8. Error in the estimated and true trajectories of a mobile cognitive radio using Kalman filter with the ESRB localization scheme.

\section{CONCLUSION}

The focus of this paper is how to track the position and velocity of a mobile $\mathrm{CR}$ over time using the collaborative spectrum sensing results of a WSN in conjunction with a recursive estimator. The ESRB localization scheme is modified to incorporate the Kalman filter as a recursive estimator to track a CR. The proposed modification estimates not only the position and velocity of a $\mathrm{CR}$ over time, but also reduce the communication overhead and storage requirements within the WSN resulting in a more efficient scheme.

\section{REFERENCES}

[1] A. Adams, M. Tummala, and J. McEachen, "Source localization in a cognitive radio environment consisting of frequency and spatial mobility," in World of Wireless, Mobile and Multimedia Networks (WoWMoM), 2012 IEEE International Symposium on a, 2012, pp. 1-6.
[2] R. D. Hinman, "Application of cognitive radio technology to legacy military waveforms in a JTRS (joint tactical radio system) radio," in IEEE Military Communications Conference, 2006. MILCOM 2006. IEEE, Oct. 2006, pp. 1-5.

[3] W. Wang, Z. Zhang, and A. Huang, "Spectrum aggregation: Overview and challenges," Network Protocols and Algorithms, vol. 2, no. 1, pp. 184-196, May 2010. [Online]. Available: http://macrothink.org/journal/index.php/npa/article/view/329

[4] D. Gurney, G. Buchwald, L. Ecklund, S. L. Kuffner, and J. Grosspietsch, "Geo-location database techniques for incumbent protection in the TV white space," in 3rd IEEE Symposium on New Frontiers in Dynamic Spectrum Access Networks, 2008. DySPAN 2008. IEEE, Oct. 2008, pp. 1-9.

[5] C. Stevenson, C. Cordeiro, E. Sofer, and G. Chouinard, "Functional requirements for the 802.22 WRAN standard.doc," Nov. 2006. [Online]. Available: https://mentor.ieee.org/802.22/file/05/22-05-000748-0000-draft-wranrqmts- doc.doc

[6] Y. Zhao, L. Morales, J. Gaeddert, K. K. Bae, J.-S. Um, and J. H. Reed, "Applying radio environment maps to cognitive wireless regional area networks," in 2nd IEEE International Symposium on New Frontiers in Dynamic Spectrum Access Networks, 2007. DySPAN 2007. IEEE, Apr. 2007, pp. 115-118.

[7] Z. Wang, Z. Feng, J. Song, Y. Hu, and P. Zhang, "A practical semi range-based localization algorithm for cognitive radio," in Vehicular Technology Conference (VTC 2010-Spring), 2010 IEEE 71st. IEEE, May 2010, pp. 1-5.

[8] Z. Ma, W. Chen, K. B. Letaief, and Z. Cao, "A semi range-based iterative localization algorithm for cognitive radio networks," IEEE Transactions on Vehicular Technology, vol. 59, no. 2, pp. 704-717, Feb. 2010.

[9] V. Sonmezer, M. Tummala, J. McEachen, and A. Adams, "Cooperative wideband spectrum sensing using radio frequency sensor networks," in 2010 Conference Record of the Forty Fourth Asilomar Conference on Signals, Systems and Computers (ASILOMAR). IEEE, Nov. 2010, pp. 951-955.

[10] C. T. Kelley, Iterative methods for optimization. SIAM, 1999.

[11] J. Ma, G. Zhao, and Y. Li, "Soft combination and detection for cooperative spectrum sensing in cognitive radio networks," IEEE Transactions on Wireless Communications, vol. 7, no. 11, pp. 4502-4507, Nov. 2008.

[12] R. KALMAN, "A new approach to linear filtering and prediction problems1," Transactions of the ASME-Journal of Basic Engineering, vol. 82, pp. 35-45, 1960. [Online]. Available: http://unitedthc.com/DSP/Kalman1960.pdf

[13] D. Cavalcanti and M. Ghosh, "Cognitive radio networks: Enabling new wireless broadband opportunities," in 3rd International Conference on Cognitive Radio Oriented Wireless Networks and Communications, 2008. CrownCom 2008. IEEE, May 2008, pp. 1-6.

[14] C. Cordeiro, K. Challapali, D. Birru, S. Shankar et al., "IEEE 802.22: an introduction to the first wireless standard based on cognitive radios," Journal of communications, vol. 1, no. 1, p. 3847, 2006. 